\title{
STABILITY ANALYSIS IN TERMS OF TWO MEASURES FOR DYNAMIC SYSTEMS ON TIME SCALES'
}

\author{
BILLÛR KAYMAKÇALAN ${ }^{2}$ \\ Middle East Technical University \\ Department of Mathematics \\ Ankara 06531 TURKEY
}

\begin{abstract}
Using the theory of Lyapunov's second method developed earlier for time scales, we extend our stability results to two measures which give rise to unification of several stability concepts in a single set up.

Key words: Two measure stability, time scales, $\left(h_{0}, h\right)-L^{1}$ stability, finite interval $\left(h_{0}, h\right)-L^{1}$-stability.
\end{abstract}

AMS (MOS) subject classifications: $\quad 34 \mathrm{D} 20,34 \mathrm{C} 35$.

\section{INTRODUCTION}

It is well-known [9] that the development of stability theory in terms of two measures unifies and includes a variety of known concepts of stability in a single set up.

In [5] we have developed Lyapunov's second method in the framework of general comparison principle for dynamic systems on time scales.

In this paper we extend the above mentioned results to obtain stability analysis in terms of two measures by using the comparison result, Theorem 2.1, given in [5], in terms of Lyapunov-like functions. We further give examples for various stability concepts in terms of two measures.

We also introduce the concepts of $\left(h_{0}, h\right)-L^{1}$-stability and finite interval $\left(h_{0}, h\right)-L^{1}$-stability and give various stability criteria in terms of these notions. We further give examples illustrating the relation between these new definitions.

\footnotetext{
${ }^{1}$ Received: June, 1993. Revised: October, 1993.

${ }^{2}$ This work was partially supported by the Scientific and Technical Research Council of Turkey (TBAG-Ç2).
} 


\section{PRELIMINARIES}

Consider the dynamic system

$$
x^{\Delta}=f(t, x), x\left(t_{0}\right)=x_{0}, t_{0} \geq 0,
$$

where $f \in C_{r d}\left[\mathbb{T}^{k} \times \mathbb{R}^{n}, \mathbb{R}^{n}\right]$. Suppose that the function $f$ is smooth enough to guarantee existence, uniqueness and $r d$-continuous dependence of solutions $x(t)=x\left(t, t_{0}, x_{0}\right)$ of (1.1). Let us first define the following classes of functions for future use:

$\mathscr{S}=\left\{a \in C\left[\mathbb{R}_{+}, \mathbb{R}_{+}\right]: a(u)\right.$ is strictly increasing in $u$ and $\left.a(0)=0\right\}$,

$\mathcal{L}=\left\{\sigma \in C\left[\mathbb{R}_{+}, \mathbb{R}_{+}\right]: \sigma(u)\right.$ is strictly decreasing in $u$ and $\left.\lim _{u \rightarrow \infty} \sigma(u)=0\right\}$,

eYG $=\left\{a \in C_{r d}\left[\mathbb{T}^{k} \times \mathbb{R}_{+}, \mathbb{R}_{+}\right]: a(t, s) \in \mathscr{W}\right.$ for each $\left.t\right\}$,

$\Gamma=\left\{h \in C_{r d}\left[\mathbb{T}^{k} \times \mathbb{R}^{n}, \mathbb{R}_{+}\right]: \inf h(t, x)=0\right\}$,

$\Gamma_{0}=\left\{h \in \Gamma: i n_{x} f h(t, x)=0\right.$ for each $\left.t \in \mathbb{T}^{k}\right\}$.

We now define stability concepts for the system (1.1) in terms of two measures $h_{0}, h \in \Gamma$.

Definition 1.1: The dynamic system (1.1) is said to be

$\left(S_{1}\right)\left(h_{0}, h\right)$-equistable if, for each $\epsilon>0, t_{0} \in \mathbb{T}^{k}$, there exists a positive function $\delta=\delta\left(t_{0}, \epsilon\right)$ that is $r d$-continuous in $t_{0}$ for each $\epsilon$ such that $h_{0}\left(t_{0}, x_{0}\right)<\delta$ implies $h(t, x(t))<\epsilon, t \geq t_{0}$, where $x(t)=x\left(t, t_{0}, x_{0}\right)$ is any solution of system (1.1),

$\left(S_{2}\right) \quad\left(h_{0}, h\right)$-uniformly stable if the $\delta$ in $\left(S_{1}\right)$ is independent of $t_{0}$.

Other stability concepts for the system (1.1) can be defined similarly.

Definition 1.2: Let $h_{0}, h \in \Gamma$. Then we say that

(i) $h_{0}$ is finer than $h$ if there exists a $\rho>0$ and a function $\varphi \in \mathbb{C S}$ such that $h_{0}(t, x)<\rho$ implies $h(t, x) \leq \varphi\left(t, h_{0}(t, x)\right)$;

(ii) $h_{0}$ is uniformly finer than $h$ if in $(i) \varphi$ is independent of $t$.

Definition 1.3: Let $V \in C_{r d}\left[\mathbb{V}^{k} \times \mathbb{R}^{n}, \mathbb{R}_{+}\right]$. Then $V$ is said to be

(i) $h$-positive definite if there exists a $\rho>0$ and a function $b \in \mathscr{K}$ such that $b(h(t, x)) \leq V(t, x)$ whenever $h(t, x)<\rho$;

(ii) $h$-decrescent if there exists a $\rho>0$ and a function $a \in \mathscr{K}$ such that $V(t, x) \leq a(h(t, x))$ whenever $h(t, x)<\rho ;$ 
(iii) $h$-weakly decrescent if there exists a $\rho>0$ and a function $a \in$ CIG such that $V(t, x) \leq a(t, h(t, x))$ whenever $h(t, x)<\rho$.

Definition 1.4: Let $V \in C_{r d}\left[T^{k} \times \mathbb{R}^{n}, \mathbb{R}_{+}\right]$and $\mu^{*}(t)$ be as in Definition 2.2 in [4]. Then we define

and

$$
D_{-} V^{\Delta}(t, x)=\lim _{\mu^{*}(t) \rightarrow 0} \inf \frac{V(t, x)-V\left(t-\mu^{*}(t), x-\mu^{*}(t) f(t, x)\right)}{\mu^{*}(t)}
$$

$$
D^{+} V^{\Delta}(t, x) \equiv \lim _{\mu^{*}(t) \rightarrow 0} \sup \frac{V\left(t+\mu^{*}(t), x+\mu^{*}(t) f(t, x)\right)-V(t, x)}{\mu^{*}(t)}
$$

If $V$ is differentiable, then $D_{-} V^{\Delta}(t, x)=D^{+} V^{\Delta}(t, x)=V^{\Delta}(t, x)$ where $V^{\Delta}(t, x)=V_{t}^{\Delta}(t, x)+V_{x}^{\Delta}(t, x) \cdot f(t, x)$. Here $V_{t}^{\Delta}$ is considered as in Definition 2.5 in [4] and $V_{x}^{\Delta}$ is taken as the normal derivative.

Let $x(t)$ be a solution of (1.1) existing on $\left[t_{0}, \infty\right)$ and $V(t, x)$ be locally Lipschitzian in $x$. Then, given $t \geq t_{0}$, there exists a neighborhood $U$ of $(t, x(t))$ and an $L>0$ such that

$$
|V(\tau, \xi)-V(\tau, \eta)| \leq L\|\xi-\eta\| \text { for }(\tau, \xi),(\tau, \eta) \in U
$$

Choose $\mu^{*}(t)>0$ sufficiently small so that

$$
\left(t+\mu^{*}(t), x\left(t+\mu^{*}(t)\right)\right) \in U \text { and }\left(t+\mu^{*}(t), x(t)+\mu^{*}(t) f(t, x(t))\right) \in U .
$$

Then we have

$$
\begin{aligned}
& V\left(t+\mu^{*}(t), x\left(t+\mu^{*}(t)\right)\right)-V(t, x(t))=V\left(t+\mu^{*}(t), x(t)+\mu^{*}(t) f(t, x(t))\right. \\
&\left.+\mu^{*}(t) \epsilon\right)-V(t, x(t)) \\
& \leq V\left(t+\mu^{*}(t), x(t)+\mu^{*}(t) f(t, x(t))\right)+L \mu^{*}(t)|\epsilon|-V(t, x(t)),
\end{aligned}
$$

where $\epsilon$ tends to zero with $\mu^{*}(t)$. It then follows that

$$
\begin{gathered}
\lim _{\mu^{*}(t) \rightarrow 0^{+}} \sup \frac{1}{\mu^{*}(t)}\left[V\left(t+\mu^{*}(t), x\left(t+\mu^{*}(t)\right)\right)-V(t, x(t))\right] \\
\leq \lim _{\mu^{*}(t) \rightarrow 0^{+}} \sup \frac{1}{\mu^{*}(t)}\left[V\left(t+\mu^{*}(t), x(t)+\mu^{*}(t) f(t, x(t))\right)-V(t, x(t))\right] .
\end{gathered}
$$

On the other hand, we have

$$
\begin{gathered}
\left.V\left(t+\mu^{*}(t), x\left(t+\mu^{*}(t)\right)\right)-V(t, x(t))\right) \\
\geq V\left(t+\mu^{*}(t), x(t)+\mu^{*}(t) f(t, x(t))\right)-L \mu^{*}(t)|\epsilon|-V(t, x(t))
\end{gathered}
$$


which implies

$$
\begin{gathered}
\lim _{\mu^{*}(t) \rightarrow 0^{+}} \sup \frac{1}{\mu^{*}(t)}\left[V\left(t+\mu^{*}(t), x\left(t+\mu^{*}(t)\right)\right)-V(t, x(t))\right] \\
\geq \lim _{\mu^{*}(t) \rightarrow 0^{+}} \sup \frac{1}{\mu^{*}(t)}\left[V\left(t+\mu^{*}(t), x(t)+\mu^{*}(t) f(t, x(t))\right)-V(t, x(t))\right] .
\end{gathered}
$$

Thus we obtain

$$
\begin{gathered}
\lim _{\mu^{*}(t) \rightarrow 0^{+}} \sup \frac{1}{\mu^{*}(t)}\left[V\left(t+\mu^{*}(t), x\left(t+\mu^{*}(t)\right)\right)-V(t, x(t))\right] \\
=\lim _{\mu^{*}(t) \rightarrow 0^{+}} \sup \frac{1}{\mu^{*}(t)}\left[V\left(t+\mu^{*}(t), x(t)+\mu^{*}(t) f(t, x(t))\right)-V(t, x(t))\right] .
\end{gathered}
$$

Similarly, we can show

$$
\begin{gathered}
\lim _{\mu^{*}(t) \rightarrow 0^{+}} \inf \frac{1}{\mu^{*}(t)}\left[V\left(t+\mu^{*}(t), x\left(t+\mu^{*}(t)\right)\right)-V(t, x(t))\right] \\
=\lim _{\mu^{*}(t) \rightarrow 0^{+}} \inf \frac{1}{\mu^{*}(t)}\left[V\left(t+\mu^{*}(t), x(t)+\mu^{*}(t) f(t, x(t))\right)-V(t, x(t))\right] .
\end{gathered}
$$

The following result is useful in the subsequent discussions.

Lemma 1.1: Suppose $m(t)$ is rd-continuous on $(a, b)$. Then $m(t)$ is nondecreasing (nonincreasing) on $(a, b)$ if and only if $D^{+} m^{\Delta}(t) \geq 0(\leq 0)$ for every $t \in(a, b)$, where

$$
D^{+} m^{\Delta}(t)=\lim _{\mu^{*}(t) \rightarrow 0^{+}} \sup \frac{1}{\mu^{*}(t)}\left[m\left(t+\mu^{*}(t)\right)-m(t)\right] .
$$

Proof: The condition is obviously necessary. Let us prove that it is sufficient. Assume first that $D^{+} m^{\Delta}(t)>0$ on $(a, b)$. If there exist two points $\alpha, \beta \in(a, b), \quad \alpha<\beta, \quad$ such that $m(\alpha)>m(\beta)$, then there is a $\mu$ with $m(\alpha)>\mu>m(\beta)$ and some points $t \in[\alpha, \beta]$ such that $m(t)>\mu$. Let $\xi=\sup \{t ; m(t)>\mu, t \in[\alpha, \beta]\}$. Clearly, $\xi \in(\alpha, \beta)$ and $m(\xi)=\mu$. Therefore, for every $t \in(\xi, \beta)$, we have

$$
\frac{m(t)-m(\xi)}{t-\xi}<0
$$

which implies $D^{+} m^{\Delta}(\xi) \leq 0$. This is a contradiction.

Assume now, as in the statement of the lemma, that $D^{+} m^{\Delta}(t) \geq 0$ on $(a, b)$. For any $\epsilon>0$, one gets $D^{+}[m(t)+\epsilon t]^{\Delta}=D^{+} m^{\Delta}(t)+\epsilon>0$. Hence from 
the above discussion $m(t)+\epsilon t$ is nondecreasing on $(a, b)$. Since this is true for any $\epsilon, m(t)$ is also nondecreasing on $(a, b)$. Similarly, one can prove that $m(t)$ is nonincreasing if $D^{+} m^{\Delta}(t) \leq 0$. Thus the proof of the lemma is complete.

\section{BASIC LYAPUNOV THEORY IN TERMS OF TWO MEASURES}

In general, in Lyapunov's second method, a Lyapunov function with continuous first order partial derivatives is considered. Here, we shall consider a more general case and develop the basic Lyapunov theory in terms of notions introduced in Section 1.

Theorem 2.1: Assume that

(i) $\quad V \in C_{r d}\left[\mathbb{T}^{k} \times \mathbb{R}^{n}, \mathbb{R}_{+}\right], h \in \Gamma, V(t, x)$ is locally Lipschitzian in $x$ and $h$ positive definite;

(ii) $D^{+} V^{\Delta}(t, x) \leq 0, \quad(t, x) \in S(h, \rho), \quad$ where $\quad S(h, \rho)=\left\{(t, x) \in \mathbb{T}^{k} \times \mathbb{R}^{n}\right.$ : $h(t, x)<\rho, \rho>0\}$.

Then

$(A)$ if, in addition, $h_{0} \in \Gamma, h_{0}$ is finer than $h$ and $V(t, x)$ is $h_{0}$-weakly decrescent, then the system $(1.1)$ is $\left(h_{0}, h\right)$-equistable,

$(B)$ if, in addition, $h_{0} \in \Gamma, h_{0}$ is uniformly finer than $h$, and $V(t, x)$ is $h_{0^{-}}$ decrescent, then the system (1.1.) is $\left(h_{0}, h\right)$-uniformly stable.

Proof: Let us first prove $(A)$. Since $V(t, x)$ is $h_{0}$-weakly decrescent, then for $t \in \mathbb{T}^{k}, x_{0} \in \mathbb{R}^{n}$, there exist a constant $\delta_{0}=\delta_{0}\left(t_{0}\right)>0$ and a function $a \in$ eFs such that

$$
V\left(t_{0}, x_{0}\right) \leq a\left(t_{0}, h_{0}\left(t_{0}, x_{0}\right)\right), \text { provided } h_{0}\left(t_{0}, x_{0}\right)<\delta_{0} .
$$

The fact that $V(t, x)$ is $h$-positive definite implies that there exist a constant $\rho_{0} \in(0, \rho)$ and a function $b \in \mathscr{K}$ such that

$$
b(h(t, x)) \leq V(t, x), \text { whenever } h(t, x) \leq \rho_{0} .
$$

Also, by the assumption that $h_{0}$ is finer than $h$, there exist a constant $\delta_{1}=\delta_{1}\left(t_{0}\right)>0$ and a function $\varphi \in \mathrm{C} \mathscr{S}$ such that

$$
h\left(t_{0}, x_{0}\right) \leq \varphi\left(t_{0}, h_{0}\left(t_{0}, x_{0}\right)\right), \text { if } h_{0}\left(t_{0}, x_{0}\right)<\delta_{1},
$$

where $\delta_{1}$ is chosen so that $\varphi\left(t_{0}, \delta_{1}\right)<\rho_{0}$. 
Let $\epsilon \in\left(0, \rho_{0}\right)$ and $t_{0} \in \mathbb{T}^{k}$ be given. By the assumption on $a$, there exists a $\delta_{2}=\delta_{2}\left(t_{0}, \epsilon\right)>0$ that is $r d$-continuous in $t_{0}$ such that

$$
a\left(t_{0}, \delta_{2}\right)<b(\epsilon) \text {. }
$$

Choose $\delta\left(t_{0}\right)=\min \left\{\delta_{0}, \delta_{1}, \delta_{2}\right\}$. Then $h_{0}\left(t_{0}, x_{0}\right)<\delta$ implies, by (2.1)-(2.4), that $b\left(h\left(t_{0}, x_{0}\right)\right) \leq V\left(t_{0}, x_{0}\right) \leq a\left(t_{0}, h_{0}\left(t_{0}, x_{0}\right)\right)<b(\epsilon)$, which in turn yields that $h\left(t_{0}, x_{0}\right)<\epsilon$. We now claim that for every solution $x(t)=x\left(t, t_{0}, x_{0}\right)$ of (1.1) with $h_{0}\left(t_{0}, x_{0}\right)<\delta$

$$
h(t, x(t))<\epsilon, t \geq t_{0} .
$$

If this is not true, then there would exist a $t_{1}>t_{0}$ such that

$$
h\left(t_{1}, x\left(t_{1}\right)\right) \geq \epsilon \text { and } h(t, x(t))<\epsilon, t \in\left[t_{0}, t_{1}\right),
$$

for some solution $x(t)=x\left(t, t_{0}, x_{0}\right)$ of (1.1). Set $m(t)=V(t, x(t))$ for $t \in\left[t_{0}, t_{1}\right]$. Since $V(t, x)$ is locally Lipschitzian in $x$, it follows from (1.4) and assumption (ii) that $D^{+} m^{\Delta}(t) \leq 0$, which implies, by Lemma 1.1, that $m(t)$ is nonincreasing in $\left[t_{0}, t_{1}\right]$. Thus it follows from (2.1)-(2.4) that

$$
b(\epsilon) \leq b\left(h\left(t_{1}, x\left(t_{1}\right)\right) \leq V\left(t_{1}, x\left(t_{1}\right)\right) \leq V\left(t_{0}, x_{0}\right)<b(\epsilon),\right.
$$

which is a contradiction. Hence $(2.5)$ is true and the system $(1.1)$ is $\left(h_{0}, h\right)$ equistable.

To prove $(B)$, note that if $V(t, x)$ is $h_{0}$-decrescent and $h_{0}$ is uniformly finer than $h$, then the functions $a$ and $\varphi$ in (2.1) and (2.3) are independent of $t$. Consequently, it is easily seen that the constant $\delta$ can be chosen to be independent of $t_{0}$. Hence the system (1.1) is $\left(h_{0}, h\right)$-uniformly stable.

We next prove a result on $\left(h_{0}, h\right)$-uniform asymptotic stability.

Theorem 2.2: Assume that

(i) $h_{0}, h \in \Gamma$ and $h_{0}$ is uniformly finer than $h$,

(ii) $V \in C_{r d}\left[\mathbb{V}^{k} \times \mathbb{R}^{n}, \mathbb{R}_{+}\right], V(t, x)$ is locally Lipschitzian in $x$, $h$-positive definite, $h_{0}$-decrescent and

$$
D^{+} V^{\Delta}(t, x) \leq-C\left(h_{0}(t, x)\right),(t, x) \in S(h, \rho), C \in \mathscr{K} .
$$

Then the system $(1.1)$ is $\left(h_{0}, h\right)$-uniformly asymptotically stable. 
Proof: Since $V(t, x)$ is $h$-positive definite and $h_{0}$-decrescent, there exist constants $0<\rho_{0} \leq \rho, 0<\delta_{0}$ and functions $a, b \in \mathscr{W}$ such that

$$
b(h(t, x)) \leq V(t, x),(t, x) \in S\left(h, \rho_{0}\right)
$$

and

$$
V(t, x) \leq a\left(h_{0}(t, x)\right), \text { if } h_{0}(t, x)<\delta_{0}
$$

It follows from Theorem 2.1 that the system $(1.1)$ is $\left(h_{0}, h\right)$-uniformly stable. Thus we let $\epsilon=\rho_{0}$, there exists $\delta_{1}=\delta_{1}(\rho)>0$ such that

$$
h_{0}\left(t_{0}, x_{0}\right)<\delta_{1} \text { implies } h(t, x(t))<\rho_{0}, t \geq t_{0},
$$

where $x(t)=x\left(t, t_{0}, x_{0}\right)$ is any solution of (1.1).

Let $0<\epsilon<\rho_{0}$ and $\delta=\delta(\epsilon)$ be the same $\delta$ as in Definition 1.1 for $\left(h_{0}, h\right)$ uniform stability. Assume that $h_{0}\left(t_{0}, x_{0}\right)<\delta^{*}=\min \left\{\delta_{0}, \delta_{1}\right\}$. Set $T=T(\epsilon)=$ $\frac{a\left(\delta^{*}\right)}{C(\delta)}+1$. To prove $\left(h_{0}, h\right)$-uniform asymptotic stability, it is enough to show that there exists a $t^{*} \in\left[t_{0}, t_{0}+T\right]$, such that

$$
h_{0}\left(t^{*}, x\left(t^{*}\right)\right)<\delta .
$$

If this is not true, then there exists a solution $x(t)=x\left(t, t_{0}, x_{0}\right)$ of (1.1) with $h_{0}\left(t_{0}, x_{0}\right)<\delta^{*}$ such that

$$
h_{0}(t, x(t)) \geq \delta, t \in\left[t_{0}, t_{0}+T\right] .
$$

Let $m(t)=V(t, x(t))$. Then it follows from condition (ii) that

$$
D^{+} m^{\Delta}(t) \leq-C\left(h_{0}(t, x(t))\right), t \geq t_{0}
$$

which implies by (2.8) that

$$
\int_{t_{0}}^{t_{0}+T} C\left(h_{0}(s, x(s))\right) \Delta s \leq m\left(t_{0}\right) \leq a\left(\delta^{*}\right) .
$$

On the other hand, from (2.9), we obtain

$$
\int_{t_{0}}^{t_{0}+T} C\left(h_{0}(s, x(s))\right) \Delta s \geq C(\delta) T>a\left(\delta^{*}\right),
$$

which is a contradiction. Thus the proof of the theorem is complete. 
Next, we discuss some examples, as applications of the above results.

Example 2.1: Consider the dynamic system

$$
\left\{\begin{array}{c}
x_{1}^{\Delta}=-\frac{1}{2} x_{1}+x_{2}-x_{3}, \\
x_{2}^{\Delta}=-e^{t} x_{1} \\
x_{3}^{\Delta}=\left(x_{2}-x_{3}\right)^{2} e^{t} .
\end{array}\right.
$$

Choose $V(t, x)=x_{1}^{2} e^{t}+\left(x_{2}-x_{3}\right)^{2}, \quad h(t, x)=\left|x_{1}\right|$ and $h_{0}(t, x)=\sqrt{x_{1}^{2}+x_{2}^{2}+x_{3}^{2}}$. Then we have

$$
(h(t, x))^{2} \leq V(t, x) \leq 2 e^{t}\left(h_{0}(t, x)\right)^{2}, \quad(t, x) \in \mathbb{T}^{k} \times \mathbb{R}^{3},
$$

and

$$
D^{+} V^{\Delta}(t, x)=-2 e^{t}\left(x_{2}-x_{3}\right)^{2} \leq 0, \quad(t, x) \in \mathbb{T}^{k} \times \mathbb{R}^{3} .
$$

By Theorem 2.1, we conclude that the system $(2.10)$ is $\left(h_{0}, h\right)$-equistable.

Example 2.2: Consider the dynamic system

$$
\left\{\begin{array}{l}
x^{\Delta}=-y+\left(1-x^{2}-y^{2}\right) x e^{-t} \\
y^{\Delta}=x+\left(1-x^{2}-y^{2}\right) y \sin x^{2}
\end{array}\right.
$$

Let $V(x, y)=\left(x^{2}+y^{2}-1\right)^{2}, h_{0}=h=\left|x^{2}+y^{2}-1\right|$. Then we see

$$
h^{2}(x, y) \leq V(x, y) \leq h_{0}^{2}(x, y),(x, y) \in \mathbb{R}^{2},
$$

and

$$
D^{+} V^{\Delta}(x, y)=-4\left(x^{2}+y^{2}-1\right)^{2}\left(x^{2} e^{-t}+y^{2} \sin x^{2}\right) \leq 0,(t, x, y) \in \mathbb{T}^{k} \times \mathbb{R}^{2} .
$$

Thus $\left(h_{0}, h\right)$-uniform stability follows from Theorem 2.1 .

Example 2.3: Consider the dynamic system

$$
\left\{\begin{array}{c}
x_{1}^{\Delta}=-x_{1}\left(1+\sin ^{2} x_{3}\right)-2 x_{2} e^{-t} \\
x_{2}^{\Delta}=2 x_{1}-x_{2} e^{t} \\
x_{3}^{\Delta}=-x_{1} e^{-t}+x_{2} \cos t+x_{3} \sin t .
\end{array}\right.
$$

Set $\quad V(t, x)=x_{1}^{2}+x_{2}^{2} e^{-t}, \quad h(t, x)=d(x, B) \quad$ and $\quad h_{0}(t, x)=d(x, A) \quad$ where 
$A=\left\{\left(x_{1}, x_{2}, x_{3}\right) \in \mathbb{R}^{3} ; x_{1}=x_{2}=0\right\}, B=\left\{\left(x_{1}, x_{2}, x_{3}\right) \in \mathbb{R}^{3} ; x_{1}=0\right\}$. Clearly $A \subset B$, $h^{2}(t, x) \leq V(t, x) \leq h_{0}^{2}(t, x)$ and $D^{+} V^{\Delta}(t, x) \leq-2 h_{0}^{2}(t, x)$. Thus, by Theorem 2.2, the system $(2.12)$ is $\left(h_{0}, h\right)$-uniformly asymptotically stable.

\section{COMPARISON METHOD}

The concept of Lyapunov function together with the theory of dynamic inequalities provides a very general comparison principle under much less restrictive assumptions. In this setup, Lyapunov function may be viewed as a transformation which reduces the study of a given complicated dynamic system to the study of relatively simpler scalar dynamic equation.

Let us consider the following scalar dynamic equation

$$
u^{\Delta}=g(t, u), u\left(t_{0}\right)=u_{0} \geq 0
$$

where $g \in C_{r d}\left[\mathbb{T}^{k} \times \mathbb{R}, \mathbb{R}\right]$ and $g(t, 0) \equiv 0$.

Definition 3.1: Let $\gamma(t)$ be a solution of (3.1) existing on some interval $J=\left[t_{0}, t_{0}+\alpha\right), 0<\alpha \leq+\infty$. Then $\gamma(t)$ is said to be the maximal solution of (3.1) if for every solution $u(t)=u\left(t, t_{0}, u_{0}\right)$ of (3.1) existing on $J$, the following inequality holds

$$
u(t) \leq \gamma(t), t \in J
$$

We now refer to Theorem 2.1 in [5] for the basic comparison result in terms of Lyapunov function $V$ and having this theorem at our disposal, we can establish some sufficient conditions for the $\left(h_{0}, h\right)$-stability properties of the dynamic system (1.1).

First we need the following definition.

Definition 3.2: The trivial solution $u(t) \equiv 0$ of (5.3.1) is said to be equistable if for any $\epsilon>0$ and $t_{0} \in \mathbb{T}^{k}$, there exists a $\delta=\delta\left(t_{0}, \epsilon\right)>0$ that is $r d$ continuous in $t_{0}$ for each $\epsilon$ such that $u_{0}<\delta$ implies $u\left(t, t_{0}, u_{0}\right)<\epsilon, t \geq t_{0}$, $u\left(t, t_{0}, u_{0}\right)$ being any solution of (3.1).

Theorem 3.1: Assume that

$\left(A_{0}\right) h_{0}, h$ are $r d$-continuous, belong to the class $\Gamma$ and $h_{0}$ is uniformly finer than $h$; 
$\left(A_{1}\right) \quad V \in C_{r d}\left[\mathbb{T} \times \mathbb{R}^{n}, \mathbb{R}_{+}\right], V(t, x)$ is locally Lipschitzian in $x$, h-positive definite and $h_{0}$-decrescent;

$\left(A_{2}\right) \quad g \in C_{r d}\left[\mathbb{T} \times \mathbb{R}_{+}, \mathbb{R}\right]$ and $g(t, 0) \equiv 0$;

$\left(A_{3}\right) \quad D_{-} V^{\Delta}(t, x) \leq g(t, V(t, x)),(t, x) \in \mathbb{T} \times \mathbb{R}^{n}$.

Then the stability properties of the trivial solution of (3.1) imply the corresponding $\left(h_{0}, h\right)$-stability properties of $(1.1)$.

Proof: We shall only prove $\left(h_{0}, h\right)$-equiasymptotic stability of (1.1). For this purpose, let us first prove $\left(h_{0}, h\right)$-equistability.

Since $V$ is $h$-positive definite, there exists a $b \in \mathscr{K}$ such that

$$
b(h(t, x)) \leq V(t, x),(t, x) \in \mathbb{T} \times \mathbb{R}^{n} .
$$

Let $\epsilon>0$ and $t_{0} \in \mathbb{T}$ be given. Suppose that the trivial solution of (3.1) is equistable. Then given $b(\epsilon)>0$ and $t_{0} \in \mathbb{T}$, there exists a function $\delta_{1}=\delta_{1}\left(t_{0}, \epsilon\right)>0$ such that

$$
u_{0}<\delta_{1} \text { implies } u\left(t, t_{0}, u_{0}\right)<b(\epsilon), t \in \mathbb{T}
$$

where $u\left(t, t_{0}, u_{0}\right)$ is any solution of (3.1). We choose $u_{0}=V\left(t_{0}, x_{0}\right)$. Since $V$ is $h_{0^{-}}$ decrescent and $h_{0}$ is uniformly finer than $h$, there exists a $\lambda_{0}>0$ and a function $a \in \mathscr{T}$ such that for $\left(t_{0}, x_{0}\right) \in S\left(h_{0}, \lambda_{0}\right)$ where $S\left(h_{0}, \lambda_{0}\right)=\left\{(t, x) \in \mathbb{T} \times \mathbb{R}^{n}: h_{0}(t, x)<\right.$ $\left.\lambda_{0}, \lambda_{0}>0\right\}$, we have

$$
h\left(t_{0}, x_{0}\right)<\lambda_{0} \text { and } V\left(t_{0}, x_{0}\right) \leq a\left(h_{0}\left(t_{0}, x_{0}\right)\right) .
$$

It then follows from (3.3) that

$$
b\left(h\left(t_{0}, x_{0}\right)\right) \leq V\left(t_{0}, x_{0}\right) \leq a\left(h_{0}\left(t_{0}, x_{0}\right)\right),\left(t_{0}, x_{0}\right) \in S\left(h_{0}, \lambda_{0}\right) .
$$

Choose $\delta=\delta\left(t_{0}, \epsilon\right)$ such that $0<\delta \leq \lambda_{0}, a(\delta)<\delta_{1}$ and let $h_{0}\left(t_{0}, x_{0}\right)<\delta$. Then (3.5) shows that $h\left(t_{0}, x_{0}\right)<\epsilon$ since $\delta_{1}<b(\epsilon)$. We claim that

$$
h(t, x(t))<\epsilon, t \geq t_{0} \text { whenever } h_{0}\left(t_{0}, x_{0}\right)<\delta,
$$

where $x(t)=x\left(t, t_{0}, x_{0}\right)$ is any solution of $(1.1)$ with $h_{0}\left(t_{0}, x_{0}\right)<\delta$. If this is not true, then there exists a $t_{1}>t_{0}$ and a solution $x(t)$ of (1.1) such that

$$
\epsilon \leq h\left(t_{1}, x\left(t_{1}\right)\right) \text { and } h(t, x(t))<\epsilon, t_{0} \leq t \leq t_{1},
$$

in view of the fact that $h\left(t_{0}, x_{0}\right)<\epsilon$ whenever $h_{0}\left(t_{0}, x_{0}\right)<\delta$. Using standard 
arguments [8], we have

$$
V(t, x(t)) \leq r\left(t, t_{0}, u_{0}\right), t_{0} \leq t \leq t_{1},
$$

where $r\left(t, t_{0}, u_{0}\right)$ is the maximal solution of (3.1). Now the relations (3.3), (3.4), (5.3.7) and (3.8) yield

$$
b(\epsilon) \leq V\left(t_{1}, x\left(t_{1}\right)\right) \leq r\left(t_{1}, t_{0}, u_{0}\right)<b(\epsilon),
$$

a contradiction, proving $\left(h_{0}, h\right)$-stability of (1.1).

Suppose next that the trivial solution of (3.1) is equi-attractive. Then we have that, given $b(\epsilon)>0$ from $\left(h_{0}, h\right)$-stability and $t_{0} \in \mathbb{T}$, there exist positive numbers $\delta_{1}^{*}=\delta_{1}^{*}\left(t_{0}\right)$ and $T=T\left(t_{0}, \epsilon\right)>0$ such that

$$
u_{0}<\delta_{1}^{*} \text { implies } u\left(t, t_{0}, u_{0}\right)<b(\epsilon), t \geq t_{0}+T \text {. }
$$

Choosing $u_{0}=V\left(t_{0}, x_{0}\right)$ as before, we find a $\delta_{0}^{*}=\delta_{0}^{*}\left(t_{0}\right)>0$ such that $0<\delta_{0}^{*} \leq \lambda_{0}$ and $a\left(\delta_{0}^{*}\right)<\delta_{1}^{*}$. Let $h_{0}\left(t_{0}, x_{0}\right)<\delta_{0}$. Then the estimate (3.8) is valid for all $t \geq t_{0}$. Suppose now that there exists a sequence $\left\{t_{k}\right\}, t_{k} \geq t_{0}+T, t_{k} \rightarrow \infty$ as $k \rightarrow \infty$ such that $\epsilon \leq h\left(t_{k}, x\left(t_{k}\right)\right)$ where $x(t)$ is any solution of (1.1) such that $h_{0}\left(t_{0}, x_{0}\right)<\delta_{0}$. This leads to a contradiction

$$
b(\epsilon) \leq V\left(t_{k}, x\left(t_{k}\right)\right) \leq r\left(t_{k}, t_{0}, u_{0}\right)<b(\epsilon)
$$

because of (3.8) and (3.9). Hence the system (1.1) is $\left(h_{0}, h\right)$-equi-asymptotically stable and the proof is complete.

Remark 3.1: Usually when stability properties for differential systems are proven, one imposes conditions on $V(t, x)$ only in $\mathbb{R}_{+} \times S(\rho)$, where $S(\rho)=$ $\left[x \in \mathbb{R}^{n}:|x|<\rho\right]$, because stability notions are of local nature relative to the trivial solution. On the other hand, when we deal with difference equations, we need, either to assume $S(\rho)$ is invariant or work in the entire $\mathbb{R}^{n}$, since we have no control of how large the solutions grow being discontinuous. As a result, if $t_{1}$ in (3.7) is a scattered point, for example, $h\left(t_{1}, x\left(t_{1}\right)\right)>\epsilon$ and it may happen that $h\left(t_{1}, x\left(t_{1}\right)\right)>\rho$ if we impose condition only on $S(h, \rho)$ for some $\rho>0$ instead of $\mathbb{R}^{n}$. 


\section{4. $L^{1}$-STABILITY AND FINITE INTERVAL $L^{1}$-STABILITY}

In this section we introduce the new concepts of $L^{1}$-stability and finite interval $L^{1}$-stability in terms of two measures and extend Theorem 3.1 in this context.

Let $\mathbb{T}$ be a time scale. Then $\mathbb{T}$ is countable union of compact subsets of $\mathbb{R}_{+}$. There may be more than one way of denoting $T$ as such a union. Each component, being a compact set, is Lebesgue measurable with measure $m$. Hence $\mathbb{T}$ is Lebesgue measurable. Let $f$ be any $\mathbb{R}$ valued function on $\mathbb{T}$. Measurability of $f$ is defined with respect to measurable subsets of $T$. Lebesgue integral of $f$ over $T$ is defined in the usual way the integral over a measurable set is defined. Let $L^{1}(\mathbb{T})$ or $L^{1}$ be the usual class of absolutely summable functions on $\mathbb{T}$. Let $\mathscr{T}$ be as in Section 1 . We define,

$$
\begin{gathered}
\mathscr{G}^{1}=\left\{b \in \mathscr{G}: \text { for any } u: \mathbb{T} \rightarrow \mathbb{R}_{+}, u \in L^{1}\right. \text { implies, } \\
\left.\qquad\left(b^{-1} u\right): \mathbb{T} \rightarrow \mathbb{R}_{+} \text {is in } L^{1}\right\} .
\end{gathered}
$$

$\left(\right.$ Here $\left(b^{-1} u\right)(t)=b^{-1}(u(t))=\alpha$ such that $\left.b(\alpha)=u(t)\right)$.

Let $h_{0}, h \in \Gamma$ be two measures as usual, with $h_{0}$ being finer than $h$.

Definition 4.1: The trivial solution of (1.1) is said to be $\left(h_{0}, h\right)-L^{1}$-stable if it is $\left(h_{0}, h\right)$-equistable in the sense of $\left(S_{1}\right)$ and there exists $\delta=\delta\left(t_{0}\right)$ such that

$$
h_{0}\left(t_{0}, x_{0}\right)<\delta \text { implies } \int_{t_{0}}^{\infty} h(s, x(s)) \Delta s<\infty .
$$

Definition 4.2: Let $\left[T_{1}, T_{2}\right] \subset \mathbb{T}^{k}$ be a given interval. The trivial solution of (1.1) is said to be $\left(h_{0}, h\right)-L^{1}$-stable on $\left[T_{1}, T_{2}\right]$ (i.e. it is said to have finite interval $L^{1}$-stability) if given $\epsilon>0, t_{0}>0$, there exists $\delta=\delta\left(t_{0}, \epsilon, T_{1}, T_{2}\right)>0$ such that

$$
h_{0}\left(t_{0}, x_{0}\right)<\delta \text { implies } \int_{T_{1}}^{T_{2}} h\left(s, x\left(s, t_{0}, x_{0}\right)\right) \Delta s<\epsilon .
$$

Many times, the trivial solution may not indicate the full performance of the system. Hence we define,

Definition 4.1': The system $(1.1)$ is said to be $\left(h_{0}, h\right)-L^{1}$-stable if there exists $\delta>0$ such that 


$$
h_{0}\left(t_{0}, x_{0}\right)<\delta \text { implies } \int_{t_{0}}^{\infty} h(s, x(s)) \Delta s<\infty
$$

Definition 4.2': The system $(1.1)$ is said to have finite interval $\left(h_{0}, h\right)-L^{1}$ stability on the interval $\left[T_{1}, T_{2}\right] \subset T^{k}$ if given $\epsilon>0$, we can find a $\delta>0$ such that

$$
h_{0}\left(t_{0}, x_{0}\right)<\delta \text { implies } \int_{T_{1}}^{T_{2}} h\left(t, x\left(t, t_{0}, x_{0}\right)\right) \Delta t<\epsilon .
$$

We note that, when stability of the trivial solution of $(1.1)$ is assumed, $L^{1}$ stability on $\left[T_{1}, T_{2}\right]$ follows quite naturally. We refer to Remark 4.1 for details. Also we point out that the usual $L^{p}$-stability defined as in [7] can be obtained as a special case of $\left(h_{0}, h\right)-L^{1}$-stability by taking $h(t, x)=\|x\|_{p}$, for any $p>1$.

Theorem 4.1: Assume that $\left(A_{0}\right),\left(A_{2}\right)$ and $\left(A_{3}\right)$ of Theorem 3.1 remain valid with $\left(A_{1}\right)$ replaced by

$\left(A_{1}\right)^{*} V \in C_{r d}\left[\mathbb{T} \times \mathbb{R}^{n}, \mathbb{R}_{+}\right], V(t, x)$ is locally Lipschitzian in $x$ and h-positive definite.

Then, if the trivial solution of $(3.1)$ is $\left(h_{0}, h\right)-L^{1}$-stable, we have that the trivial solution of $(1.1)$ is also $\left(h_{0}, h\right)-L^{1}$-stable.

Proof: Under the hypothesis, by Theorem 3.1 it follows that the trivial solution of $(1.1)$ is $\left(h_{0}, h\right)$-equistable. Further, we have by (3.8)

$$
b(h(t, x(t))) \leq V(t, x(t)) \leq r\left(t, t_{0}, u_{0}\right)
$$

where $r\left(t, t_{0}, u_{0}\right)$ is the solution of

$$
u^{\Delta}=g(t, u), u\left(t_{0}\right)=V\left(t_{0}, x_{0}\right)
$$

Since by hypothesis $r\left(t, t_{0}, u_{0}\right) \in L^{1}$, as a result of the $L^{1}$-stability of scalar equations and $b \in \mathscr{K}^{1}$, it then follows that

$$
\int_{t_{0}}^{\infty} h(t, x(t))^{p} \Delta t \leq \int_{t_{0}}^{\infty}\left(b^{-1} r(t)\right) \Delta t<\infty .
$$

This proves the $\left(h_{0}, h\right)-L^{1}$-stability of the trivial solution of $(1.1)$.

Remark 4.1: Let $\epsilon>0, T_{1}, T_{2} \in \mathbb{T}^{k}$ with $T_{2}>T_{1}$ and $m\left(\left[T_{1}, T_{2}\right]\right)>0$ where $m$ is the Lebesgue measure.

Choose $\epsilon^{\prime}>0$ such that $b^{-1}\left(\epsilon^{\prime}\right)<\left(\frac{\epsilon}{m\left(\left[T_{1}, T_{2}\right]\right)}\right)$. This is possible since $b^{-1}$ 
is continuous at 0 .

Since the zero solution of the scalar equation (3.1) is stable, there exists $\delta^{\prime}>0$ such that

$$
r\left(t, t_{0}, u_{0}\right)<\epsilon^{\prime} \text { whenever } 0<u_{0}<\delta^{\prime} .
$$

Since $V(t, x)<r\left(t, t_{0}, V\left(t_{0}, x_{0}\right)\right)$, using the $r d$-continuity of $V$ at $\left(t_{0}, x_{0}\right)$, choose $\delta>0$ such that $\left|x_{0}\right|<\delta$ implies $V\left(t_{0}, x_{0}\right)<\delta^{\prime}$. Then we have,

$$
b\left(h\left(t, x\left(t, t_{0}, x_{0}\right)\right)\right) \leq V\left(t, x\left(t, t_{0}, x_{0}\right)\right)<r\left(t, t_{0}, x_{0}\right)<\epsilon^{\prime} .
$$

Hence,

Thus

$$
\begin{gathered}
h\left(t, x\left(t, t_{0}, x_{0}\right)\right)<b^{-1}\left(\epsilon^{\prime}\right), t \in\left[T_{1}, T_{2}\right] \\
<\left(\frac{\epsilon}{m\left(\left[T_{1}, T_{2}\right]\right)}\right) .
\end{gathered}
$$

$$
\int_{\left[T_{1}, T_{2}\right]} h \Delta t<\frac{\epsilon}{m\left(\left[T_{1}, T_{2}\right]\right)} \int_{\left[T_{1}, T_{2}\right]} \Delta t=\epsilon .
$$

Hence, finite interval $\left(h_{0}, h\right)-L^{1}$-stability is trivial if we assume the stability of the zero solution of (1.1). So, $\left(h_{0}, h\right)-L^{1}$-stability of the trivial solution, as defined earlier, implies equistability of the trivial solution which in turn implies the finite interval $\left(h_{0}, h\right)-L^{1}$-stability. However,

\section{Remark 4.2:}

(i-) $\left(h_{0}, h\right)-L^{1}$-stability of a system need not imply finite interval $\left(h_{0}, h\right)$ $L^{1}$-stability of the system.

To see this, let us consider the following example.

Let $f$ be a differentiable function on $[0, \infty)$ such that $f(t) \geq \epsilon>0$ on $\left[t_{1}, t_{2}\right]$ and $f$ is integrable on $\left[t_{2}, \infty\right)=\left[0, t_{1}\right)^{c} \cap \mathbb{T}^{k}$. Consider the equation

$$
x^{\Delta}(t)=\left\{\begin{array}{cc}
f^{\Delta}(t)-\frac{x(t)-f(t)}{t_{1}-t}, & 0 \leq t<t_{1} \\
f^{\Delta}(t), & t \geq t_{1} .
\end{array}\right.
$$

This has the solution,

$$
x(t)=\left\{\begin{array}{cc}
f(t)+\left(t_{1}-t\right) x_{0}, & t<t_{1} \\
f(t), & t \geq t_{1} .
\end{array}\right.
$$


Hence

$$
\int_{0}^{\infty} x(t) \Delta t=\int_{0}^{t_{1}}\left(f(s)+\left(t_{1}-s\right) x_{0}\right) \Delta s+\int_{t_{1}}^{\infty} f(s) \Delta s<\infty
$$

since the first integral is over $\left[0, t_{1}\right]$ and $f$ is assumed to be integrable.

Hence the trivial solution of (4.1) is $\left(h_{0}, h\right)-L^{1}$-stable with $h_{0}(t, x)=h(t, x)=\|x\|$. In fact, the region of $L^{1}$-stability is $\mathbb{R}$, so global stability follows.

However, since $f(t)>\epsilon$ on $\left[t_{1}, t_{2}\right]$,

$$
\int_{t_{1}}^{t_{2}} x(t) \Delta t>\epsilon\left(t_{2}-t_{1}\right)
$$

irrespective of the choice of $x_{0}$.

Hence the system (4.1) is not $\left[t_{1}, t_{2}\right]-L^{1}$-stable. This is quite natural if the influence of the initial data vanishes in a finite time.

(ii-) The obvious advantage of finite interval $L^{1}$-stability is that even when the trivial solution of the system is unstable in the usual sense, it may be finite interval $L^{1}$-stable, as can be seen by the simple example of $x^{\Delta}=a x, a>0$. Clearly, this system is not $L^{1}$-stable, in fact, not even stable, but of course the trivial solution is $L^{1}$-stable in any finite interval.

We can refine Theorem 4.1 and obtain uniformity results for the $\left(h_{0}, h\right)$ $L^{1}$-stability of the trivial solution of (1.1) based on the following definitions.

Definition 4.3: The trivial solution of (1.1) is said to be

(i) uniformly $\left(h_{0}, h\right)$ - $L^{1}$-stable if $\delta$ in Definition 4.1 is independent of $t_{0}$, and

(ii) uniformly $\left(h_{0}, h\right)$-finite interval- $L^{1}$-stable if $\delta$ in Definition 4.2 is independent of $t_{0}$.

Corollary 4.1: If all the assumptions $\left(A_{0}\right)-\left(A_{3}\right)$ of Theorem 3.1 are kept valid, including $h_{0}$-decrescentness of $V(t, x)$, then Theorem 4.1 can be extended to give the uniform $\left(h_{0}, h\right)-L^{1}$-stability of the trivial solution of (1.1) whenever the uniform $\left(h_{0}, h\right)-L^{1}$-stability of the trivial solution of (3.1) is assumed. 


\section{FINITE INTERVAL $L^{\text {p}}$-STABILITY FOR LINEAR AND WEAKLY NONLINEAR SYSTEMS}

For the linear system

$$
x^{\Delta}=A(t) x, x\left(t_{0}\right)=x_{0}
$$

let $\Phi_{A}\left(\cdot, t_{0}\right)$ be the fundamental solution guaranteed by Theorem 3.2 in [4].

We will make use of the following relation between the transition matrix $\Phi_{A}\left(t, t_{0}\right)$ and the fundamental matrix $\Phi(t)$ :

$$
\Phi_{A}\left(t, t_{0}\right)=\Phi(t) \cdot \Phi^{-1}\left(t_{0}\right) .
$$

So, by using the formula (4.3) in [6] with the above notation we have that

$$
x(t)=\Phi(t) \Phi^{-1}\left(t_{0}\right) x_{0}
$$

is the solution of the system (5.1).

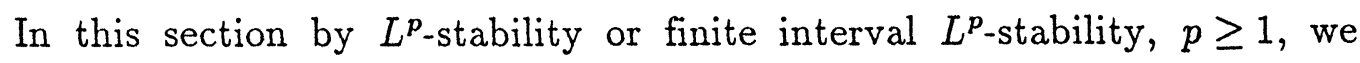
understand the corresponding $\left(h_{0}, h\right)-L^{1}$-stability concept with $h_{0}(t, x)=h(t, x)=$ $\|x\|_{p}$. We can easily observe the finite interval $-L^{p}$-stability of the trivial solution of the system (5.1) as follows:

By use of (5.3), for any $t_{1}, t_{2} \in \mathbb{T}^{k}, t_{1}<t_{2}$

$$
\begin{gathered}
\int_{t_{1}}^{t_{2}}\|x(t)\|^{p} \Delta t \leq \int_{t_{1}}^{t_{2}}\left\|\Phi(t) \Phi^{-1}\left(t_{0}\right) x_{0}\right\|^{p} \Delta t \\
\leq\left\|\Phi^{-1}\left(t_{0}\right)\right\|^{p}\left\|x_{0}\right\|^{p} \int_{t_{1}}^{t_{2}}\|\Phi(t)\|^{p} \Delta t \\
\leq C\|\Phi\|_{p}
\end{gathered}
$$

where $\left\|\Phi^{-1}\left(t_{0}\right)\right\|^{p} \cdot\left\|x_{0}\right\|^{p}=C$.

Since $\Phi$ is $r d$-continuous, when $t_{2}-t_{1}<\infty,\|\Phi\|_{p}<\infty$. Hence we have that $\int_{t_{1}}^{t_{2}}\|x(t)\|^{p} \Delta t<\infty$ and so the finite-interval- $L^{p}$-stability of the trivial solution of the system (5.1) follows easily.

We also observe that the trivial solution need not be stable. 
Next, consider the weakly perturbed system:

$$
x^{\Delta}=A(t) x+R(t, x), x\left(t_{0}\right)=x_{0} .
$$

Theorem 5.1: Let $t_{0}<t_{1}<t_{2}<\infty$ be given with $t_{0}, t_{1}, t_{2} \in \mathbb{T}^{k}$. Suppose that there exists a function $r \in L^{q}\left[t_{1}, t_{2}\right]$ with $\frac{1}{p}+\frac{1}{q}=1, p>1$, such that $R(t, 0) \equiv 0$ and

(i) $\|R(t, x)\| \leq r(t)\|x\|, \forall t \in\left[t_{1}, t_{2}\right], \forall x \in \mathbb{R}^{n}$,

(ii) $\quad\|r\|_{q}^{0,2}<\left(\|\Phi\|_{p}^{0,2}\left\|\Phi^{-1}\right\|_{\infty}^{0,2}\right)^{-1}$.

where

$$
\begin{aligned}
& \|r\|_{q}^{0,2} \equiv\left(\int_{t_{0}}^{t_{2}}|r(s)|^{q} \Delta s\right)^{1 / q} \\
& \|\Phi\|_{p}^{0,2} \equiv\left(\int_{t_{0}}^{t_{2}}\|\Phi(t)\|^{p} \Delta t\right)^{1 / p}
\end{aligned}
$$

and

with $\frac{1}{p}+\frac{1}{q}=1, p>1$.

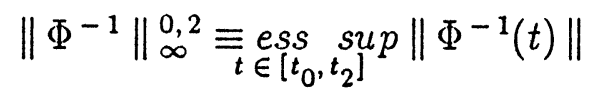

Then the trivial solution of the system (5.4) is finite-interval- $L^{p}$-stable.

Proof: The proof easily follows by the variation of parameters formula (3.7) in [6].

Using (5.2) we can rewrite (3.7) in [6] as follows:

Then

$$
x(t)=\Phi(t) \Phi^{-1}\left(t_{0}\right) x_{0}+\int_{t_{0}}^{t} \Phi(t) \Phi^{-1}(\sigma(s)) R(s, x(s)) \Delta s .
$$

$$
\begin{gathered}
\left(\int_{t_{0}}^{t_{2}}\|x(t)\|^{p} \Delta t\right)^{1 / p} \leq\left\|\Phi^{-1}\left(t_{0}\right)\right\| \cdot\left\|x_{0}\right\| \cdot\left(\int_{t_{0}}^{t_{2}}\|\Phi(t)\|^{p} \Delta t\right)^{1 / p} \\
+\left(\int_{t_{0}}^{t_{2}}\|\Phi(t)\|^{p}\left|\int_{t_{0}}^{t} \Phi^{-1}(\sigma(s)) \cdot R(s, x(s)) \Delta s\right|^{p} \Delta t\right)^{1 / p}
\end{gathered}
$$




$$
\begin{gathered}
=\left\|\Phi^{-1}\left(t_{0}\right)\right\| \cdot\left\|x_{0}\right\| \cdot\|\Phi\|_{p}^{0,2} \\
+\left(\int_{t_{0}}^{t_{2}}\|\Phi(t)\|^{p}\left|\int_{t_{0}}^{t} \Phi^{-1}(\sigma(s)) \cdot R(s, x(s)) \Delta s\right|^{p} \Delta t\right)^{1 / p}
\end{gathered}
$$

where $\|\Phi\|_{p}^{0,2}$ is defined by (5.7). Considering only the second part of the righthand side of the inequality (5.8),

$$
\begin{aligned}
& \left(\int_{t_{0}}^{t_{2}}\|\Phi(t)\|^{p}\left|\int_{t_{0}}^{t} \Phi^{-1}(\sigma(s)) \cdot R(s, x(s)) \Delta s\right|^{p} \Delta t\right)^{1 / p} \\
& \leq\|\Phi\|_{p}^{0,2} \underset{\substack{0, e s s \\
t \in\left[t_{0}, t_{2}\right]}}{\sup }\left(\int_{t_{0}}^{t}\left\|\Phi^{-1}(s) \cdot R(s, x(s))\right\| \Delta s\right) .
\end{aligned}
$$

Using condition (i) and Hölder's inequality

$$
\begin{aligned}
& \|\Phi\|_{p}^{0,2} \underset{\substack{e s s \\
t \in\left[t_{0}, t_{2}\right]}}{\operatorname{esp}}\left(\int_{t_{0}}^{t}\left\|\Phi^{-1}(s) R(s, x(s))\right\| \Delta s\right) \\
& \leq\|\Phi\|_{p}^{0,2} \underset{t \in\left[t_{0}, t_{2}\right]}{\operatorname{ess} \sup _{i}}\left\{\underset{\substack{\operatorname{ess} \sup \\
s \in\left[t_{0}, t_{2}\right]}}{\operatorname{es}}\left\|\Phi^{-1}(s)\right\| \cdot \int_{t_{0}}^{t} r(s)\|x(s)\| \Delta s\right\} \\
& \leq\|\Phi\|_{p}^{0,2} \cdot\left(\underset{t \in\left[t_{0}, t_{2}\right]}{e s s \sup }\left\|\Phi^{-1}(t)\right\|\right) \cdot\|r\|_{q}^{0,2} \cdot\left(\int_{t_{0}}^{t_{2}}\|x(s)\|^{p} \Delta s\right)^{1 / p}
\end{aligned}
$$

where $\|r\|_{q}^{0,2}$ is given by (5.5) with $\frac{1}{p}+\frac{1}{q}=1, p>1$. Now, by using (5.7) for $\left\|\Phi^{-1}\right\|_{\infty}^{0,2}$ and replacing (5.10) in (5.9), in view of (5.8) we obtain,

$$
\left[1-\left\|\Phi^{-1}\right\|_{\infty}^{0,2} \cdot\|\Phi\|_{p}^{0,2} \cdot\|r\|_{q}^{0,2}\right]\left(\int_{t_{0}}^{t_{2}}\|x(s)\|^{p} \Delta s\right)^{1 / p}
$$




$$
\leq\left\|\Phi^{-1}\left(t_{0}\right)\right\| \cdot\|\Phi\|_{p}^{0,2} \cdot\left\|x_{0}\right\|
$$

Hence,

$$
\left(\int_{t_{0}}^{t_{2}}\|x(s)\|^{p} \Delta s\right)^{1 / p} \leq \frac{\left\|\Phi^{-1}\left(t_{0}\right)\right\| \cdot\|\Phi\|_{p}^{0,2} \cdot\left\|x_{0}\right\|^{0}}{\left(1-\left\|\Phi^{-1}\right\|_{\infty}^{0,2} \cdot\|\Phi\|_{p}^{0,2} \cdot\|r\|_{q}^{0,2}\right)^{2}}
$$

Reducing the region of integration in (5.11) from $\left[t_{0}, t_{2}\right]$ to $\left[t_{1}, t_{2}\right]$ we get;

$$
\begin{gathered}
\left(\int_{t_{1}}^{t_{2}}\|x(s)\|^{p} \Delta s\right)^{1 / p} \leq\left(\int_{t_{0}}^{t_{2}}\|x(s)\|^{p} \Delta s\right)^{1 / p} \\
\leq \frac{\left\|\Phi^{-1}\left(t_{0}\right)\right\| \cdot\|\Phi\|_{p}^{0,2} \cdot\left\|x_{0}\right\|}{\left(1-\left\|\Phi^{-1}\right\|_{\infty}^{0,2} \cdot\|\Phi\|_{p}^{0,2} \cdot\|r\|_{q}^{0,2}\right)}
\end{gathered}
$$

Now by use of condition (ii) we can make the right-hand side of (5.12) as small as we want when $\left\|x_{0}\right\|<\delta$ for some $\delta>0$. Hence, the finite interval- $L^{p_{-}}$ stability of the trivial solution of the system (5.4) follows.

Remark 5.1: We note that $\left(h_{0}, h\right)-L^{1}$-stability and finite interval $L^{1}$ stability concepts are defined on time scales of positive measure. So we can not consider $L^{1}$-stability or finite interval $L^{1}$-stability on a set of Lebesgue measure zero, for instance the Cantor set. On such time scales we have to define a new measure for these stability concepts to make sense. On the other hand, the Definitions (4.1)-(4.2') still make sense even in the case of $\mathbb{T}=\mathbb{Z}$, the set of all integers, for then we can take the counting measure as the non-zero measure.

Acknowledgement: The author would like to express her gratitude to Professor S.V. Krishna for many helpful suggestions and improvements of the results.

\section{REFERENCES}

[1] Aulbach, B. and Hilger, S., A Unified Approach to Continuous and Discrete Dynamics, 53. Qualitative Theory of Differential Equations, Szeged (Hungary) 1988.

[2] Aulbach, B. and Hilger, S., Linear Dynamic Processes with Inhomogeneous Time Scales, Nonlinear Dynamics and Quantum Dynamical Systems, Akademie-Verlag, Berlin 1990. 
[3] Hilger, S., Analysis on measure chains - A unified approach to continuous and discrete calculus, Res. in Math. 18 (1990), pp. 18-56.

[4] Kaymakçalan, B., Existence and comparison results for dynamic systems on time scales, J.M.A.A. Vol. 172, No. 1, (1993), pp. 243-255.

[5] Kaymakçalan, B., Lyapunov stability theory for dynamic systems on time scales, JAMSA, Vol. 5, No. 3, pp. 275-281.

[6] Kaymakçalan, B., Linear and nonlinear variation of parameters formulae for dynamic systems on time scales, Nonlinear Differential Equations (to appear).

[7] Lakshmikantham, V., Leela, S., Differential and Integral Inequalities, Vol. I, Academic Press, New York 1969.

[8] Lakshmikantham, V., Leela, S. and Martynyuk, A.A., Stability Analysis for Nonlinear Systems, Marcel Dekker, Inc., New York 1989.

[9] Lakshmikantham, V. and Liu, X., Stability Analysis in Terms of Two Measures, World Scientific Press 1993. 


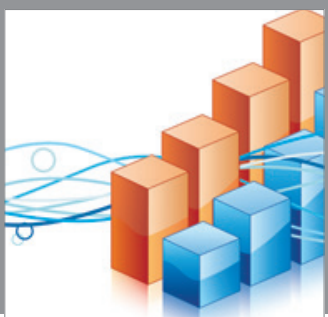

Advances in

Operations Research

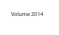

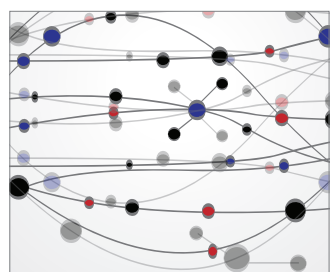

\section{The Scientific} World Journal
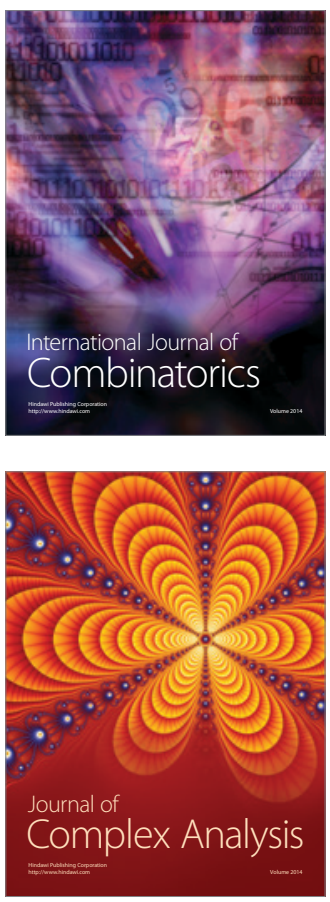

International Journal of

Mathematics and

Mathematical

Sciences
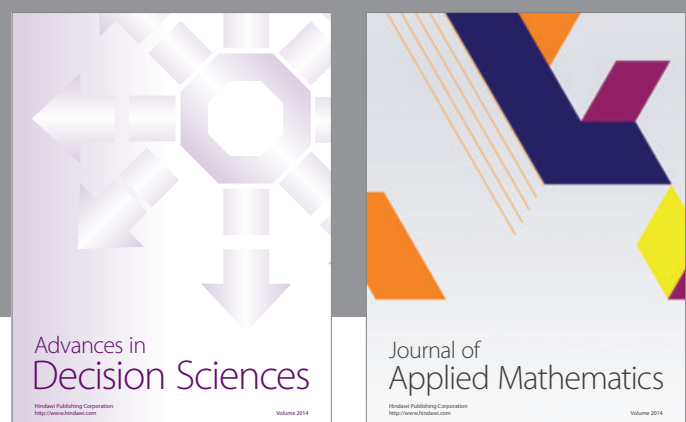

Journal of

Applied Mathematics
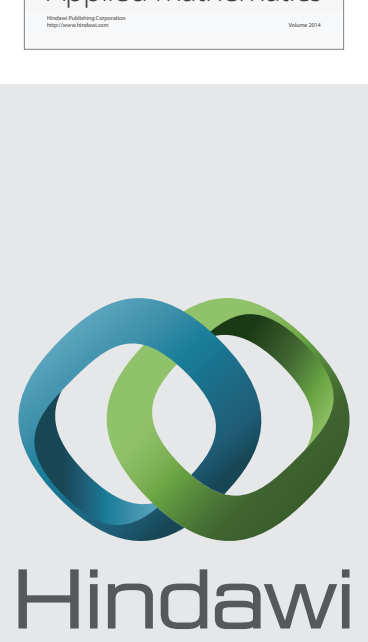

Submit your manuscripts at http://www.hindawi.com
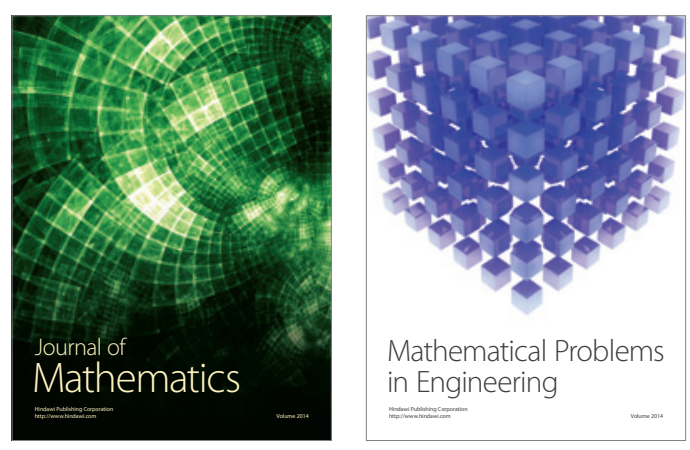

Mathematical Problems in Engineering
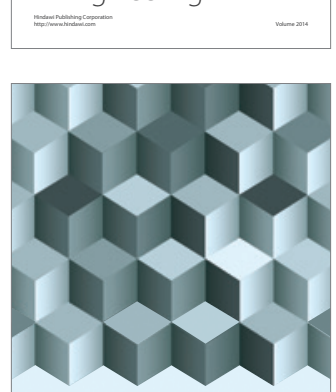

Journal of

Function Spaces
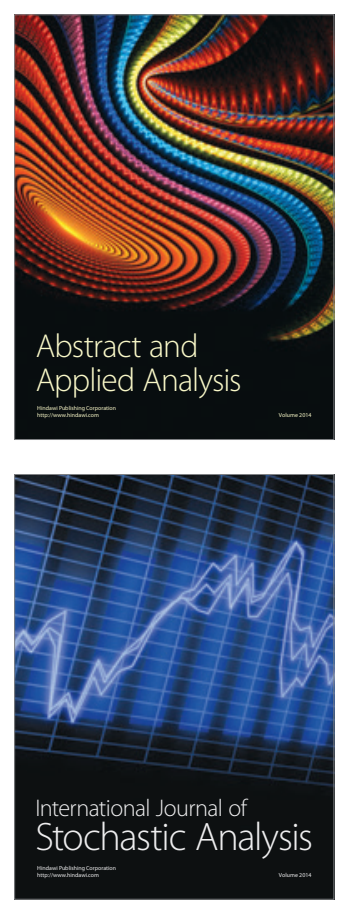

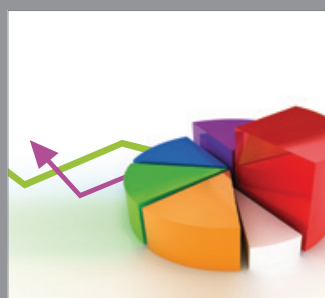

ournal of

Probability and Statistics

Promensencen
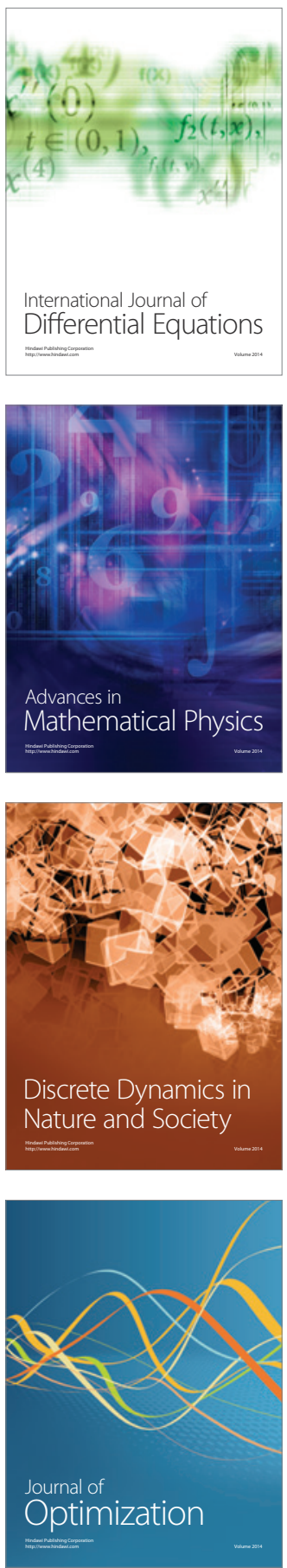